\section{BMJ Open Respiratory Research}

\title{
CPAP management of COVID-19 respiratory failure: a first quantitative analysis from an inpatient service evaluation
}

\author{
Abdul Ashish, ${ }^{1,2}$ Alison Unsworth, ${ }^{2}$ Jane Martindale, ${ }^{1,2}$ Ram Sundar, ${ }^{2}$ \\ Kanishka Kavuri, ${ }^{2}$ Luigi Sedda, ${ }^{3}$ Martin Farrier (i) ${ }^{4}$
}

To cite: Ashish A, Unsworth $A$, Martindale J, et al. CPAP management of COVID-19 respiratory failure: a first quantitative analysis from an inpatient service evaluation. BMJ Open Resp Res 2020;7:e000692. doi:10.1136/ bmjresp-2020-000692

- Additional material is published online only. To view, please visit the journal online (http://dx.doi.org/10. 1136/bmjresp-2020-000692)

Received 28 June 2020 Revised 9 0ctober 2020 Accepted 15 October 2020

\section{Check for updates}

C Author(s) (or their employer(s)) 2020. Re-use permitted under CC BY-NC. No commercial re-use. See rights and permissions. Published by BMJ.

${ }^{1}$ Edge Hill University, Ormskirk, UK

${ }^{2}$ Chest Medicine, Wrightington Wigan and Leigh NHS

Foundation Trust, Wigan, UK ${ }^{3}$ Lancaster University, Lancaster, UK

${ }^{4}$ Paediatrics, Wrightington Wigan and Leigh NHS Foundation Trust, Wigan, UK

Correspondence to Dr Martin Farrier; martin.farrier@wwl.nhs.uk

\section{ABSTRACT}

Objective To evaluate the role of continuous positive air pressure (CPAP) in the management of respiratory failure associated with COVID-19 infection. Early clinical management with limited use of CPAP ( $3 \%$ of patients) was compared with a later clinical management strategy which had a higher proportion of CPAP use (15\%).

Design Retrospective case-controlled service evaluation for a single UK National Health Service (NHS) Trust during March-June 2020 designed and conducted solely to estimate the effects of current care.

Setting The acute inpatient unit in Wrightington, Wigan and Leigh Teaching Hospitals NHS Foundation Trust, a medium-sized English NHS Trust.

Participants 206 patients with antigen confirmed COVID-19 disease and severe acute respiratory syndrome admitted between 17 March 2020 and 3 April 2020 for the early group (controls), and between 10 April 2020 and 11 May 2020 for the late group (cases). Follow-up for all cases was until 11 June by which time all patients had a final outcome of death or discharge. Both groups were composed of 103 patients. Cases and controls were matched by age and sex.

Outcome measure The outcome measure was the proportion of patients surviving at time $t$ (time from the positive result of COVID-19 test to discharge/death date). The predictors were CPAP intervention, intubation, residence in care homes and comorbidities (renal, pulmonary, cardiac, hypertension and diabetes). A stratified Cox proportional hazard for clustered data (via generalised estimating equations) and model selection algorithms were employed to identify the effect of CPAP on patients' survival and the effect on gas exchange as measured by alveolar arterial $(\mathrm{A}-\mathrm{a})$ gradient and timing of CPAP treatment on CPAP patients' survival.

Results CPAP was found to be significantly (HR 0.38 , $95 \% \mathrm{Cl} 0.36$ to 0.40 ) associated with lower risk of death in patients with hospital stay equal to, or below 7 days. However, for longer hospitalisation CPAP was found to be associated with increased risk of death (HR $1.72,95 \% \mathrm{Cl}$ 1.40 to 2.12). When CPAP was initiated within 4 days of hospital admission, the survival probability was above $73 \%$ (95\% Cl 53\% to 99\%). In addition, lower A-a gradient was associated with lower risk of death in CPAP patients (HR $1.011,95 \% \mathrm{Cl} 1.010$ to 1.013). The selected model (best fit) was stratified by sex and clustered by case/control groups. The predictors were age, intubation, hypertension

\section{Key messages}

Does ward-based continuous positive air pressure (CPAP) have a role in reducing mortality for COVID-19 associated respiratory failure?

- Early use of CPAP leads to a significant reduction in mortality.

- This study provides quantified evidence of benefit and practical supplemental information) to support the use of low cost ward based CPAP in patients with COVID-19-associated respiratory failure.

and the residency from care homes, which were found to be statistically significantly associated with patient's death/discharge.

Conclusions CPAP is a simple and cost-effective intervention. It has been established for care of other respiratory disorders but not for COVID-19 respiratory failure. This evaluation establishes that CPAP as a potentially viable treatment option for this group of patients during the first days of hospital admission. As yet there is limited availability of quantitative research on CPAP use for COVID-19. Whist this work is hampered by both the relatively small sample size and retrospective design (which reduced the ability to control potential confounders), it represents evidence of the significant benefit of early CPAP intervention. This evaluation should stimulate further research questions and larger study designs on the potential benefit of CPAP for COVID-19 infections. Globally, this potentially beneficial low cost and low intensity therapy could have added significance economically for healthcare provision in less developed countries.

\section{BACKGROUND}

COVID-19 was first reported in late 2019 and declared a pandemic on 12 March 2020. ${ }^{12}$ Prior to the COVID-19 pandemic several trials had explored the effectiveness of continuous positive air pressure (CPAP) in acute respiratory failure (ARF) providing evidence that CPAP had a role in improving important outcomes including progression 
to intubation, length of stay in intensive care unit (ICU) and/or hospital, ${ }^{3-5}$ although conflicting information emerged (eg, a recent study found that Helmet CPAP treatment failed in up to $44 \%$ of patients with moderateto-severe hypoxaemic ARF due to COVID-19 pneumonia, ${ }^{6}$ while not accounting for the timing of the CPAP intervention). However, at the beginning of the pandemic, international guidelines, including those from WHO, did not address the use of CPAP in COVID-19 patients, focusing instead on high-flow nasal oxygen (HFNO), non-invasive ventilation (NIV) and invasive mechanical ventilation once patients are intubated. In contrast, the UK National Institute for Health and Care Excellence guidance was revised on the 6 April recommending the use of CPAP although acknowledging the lack of evidence for efficacy. ${ }^{7}$ The principle concern regarding the application of CPAP guidelines centred around fears about within-ward virus contamination rather than concerns over efficacy.

The mixed information about respiratory interventions formed at the beginning of the COVID-19 pandemic had a substantial effect on the management of patients in Wrightington Wigan and Leigh Teaching Hospitals National Health Service Trust in the UK (hereafter the Trust), a medium-sized organisation caring for a population of 330000 people. Initially care of patients with respiratory failure was with early intubation and ventilation within the Intensive Care Unit. Concerns about infection control had limited plans for use of CPAP and HFNO therapies. However, as experience evolved and poor outcomes from ventilation became known, there was a greater preparedness to consider the use of CPAP. Consequently, in Mid-March, an NIV Team was set up and preparation was made to commence the use of CPAP.

There were concerns about ventilators and ICU bed availability. Intensive Care equipment became difficult to procure and CPAP machines were difficult to source. The Trust had a large stock of CPAP machines intended for sleep apnoea patients. These machines are designed to deliver air rather than an air/oxygen mix. Adjustments were made to provide microbial filtration to the exhalation port to make the machines safe to use. Protocols for CPAP delivery, ward preparation and PPE were developed and are detailed within the online supplemental file 1 .

International evidence, although qualitative, started to emerge indicating that CPAP and HFNO treatments were promising. ${ }^{8}{ }^{9}$ Data from retrospective studies in China and Italy suggested that COVID-19 patients who were hypoxaemic responded well to positive endexpiratory pressure, indicating a crucial role for NIV as a therapeutic and stopgap measure to prevent intubation. ${ }^{10}{ }^{11}$ The emergence of this evidence, aligned with growing confidence in the use of CPAP within WWL, led to a significant change in practice that is, CPAP was used earlier in the presentation of COVID-19-associated respiratory failure. The effect of early CPAP on mortality of COVID-19 patients in the Trust was audited during the first months of the UK pandemic. ${ }^{12}$ In this audit, early CPAP therapy, where CPAP is given within 48 hours of admission, and late CPAP, where CPAP is given after 48 hours were compared (although for a small sample size $n=36$ ). The earlier interventional use of CPAP was as a consequence of growing clinician confidence to begin CPAP therapy earlier and as by perceived improvement in patient responsiveness to the treatment. The study identified that those patients who commenced CPAP therapy earlier had better clinical outcomes (lower mortality) than those patients commencing CPAP after 2 days. ${ }^{12}$

In this present work, we have carried out a retrospective case-control study (within an NHS Trust service evaluation) to evaluate the differences in terms of mortality between patients admitted at the start of the pandemic to those admitted later when clinical expertise with CPAP had progressed and use had increased. Interventions (CPAP and intubation), alveolar arterial (A-a) gradient, comorbidities, residency in care homes, sex and age were included in the analyses. This service evaluation (Local ID: RD62) followed the Trust governance procedures and aimed to demonstrate and share the impact of emergent clinical expertise and service developments on patient outcomes. Due to the paucity of quantitative studies on the effect of CPAP on COVID-19 patient outcome, we also hope to encourage other service evaluations or research studies on the same question which could to further validate our findings on a larger scale.

\section{METHODS}

\section{Study design}

A retrospective case-control study within a service evaluation was undertaken. Data for all 504-19 confirmed patients hospitalised between 17 March and 18 May 2020 were used. The requirement to age and sex match patients meant that the final sample size is determined by the limitations placed by the matching process.

Patients admitted to the Trust were diagnosed with COVID-19 using PCR testing from nasal and oral swabs from the 14 March 2020. The first confirmed case was in 17 March 2020. Clinical data were collected on all positive patients to include age, sex, comorbidities, residency in care homes and clinical presentation within the Electronic Patient Record system. Retrospective identification, with randomisation, of cases and controls was carried out the 18 May 2020.

Patients were selected for CPAP by clinicians according to the unit protocol at the time. The CPAP machines were ResMed Airsense devices. These are designed to deliver CPAP as air using a pump within the CPAP machine. Oxygen was delivered at $10-15 \mathrm{~L} / \mathrm{min}$ through the face mask via a port in the face mask. This delivers an estimated 50\%-70\% fractional inspired oxygen varying according to oxygen flow and CPAP pressure.

The retrospective case-control study identified all COVID-19 patients admitted in the first 3 weeks of the epidemic (17 March-3 April 2020) creating a cohort of 103 patients (control group). Within the control cohort only three patients had received CPAP therapy. During 
the period between 3 and 10 April, there was a clinical change in the provision of CPAP with the development of ward-based CPAP service and new protocols to support the early use of CPAP. The control cohorts were matched by age and sex with 103 cases randomly selected from the last 250 patients (10 April-11 May 2020) without knowledge of their outcome. Age matching was approximate with average age difference in the matches of 0.27 years ( $\mathrm{SD} \pm 1.12$ years). For each of the 206 patients, the major comorbidities were identified and grouped in five categories: pulmonary, renal, cardiac, hypertension and diabetic. Pulmonary diagnosis was divided into three subcategories: Asthma, Bronchiectasis and Emphysema. CPAP, gas/exchange diffusion and intubation are the interventions included for this study. Gas exchange/ diffusion was assessed using an A-a gradient. This is a measure of the difference between alveolar and arterial oxygen as measured by the concentration of oxygen delivered and the arterial partial pressure of oxygen whereby an elevated value is indicative of an alveolar level respiratory illness. Finally, a binary variable (yes, no) describing residence in care homes was added. The outcome was identified as the proportion of patients surviving at time t from the COVID-19 result date, with an end point of 11 June 2020. A summary of the baseline characteristics of enrolled patients of both the early $(n=103)$ and late cohort $(\mathrm{n}=103)$ is provided in table 1.

There has been no public involvement in this study.

\section{Statistical analysis}

The statistical analysis was performed by employing a stratified Cox proportional hazards model for clustered data (SCPHC) within a model selection algorithm (ie, several models are compared and ranked by Akaike information criterion (AIC) corrected for small sample size AIC. ${ }^{13}$ Despite accounting for all the variables which are believed to be clinically important, this does not guarantee to provide the best model fitting (some variables may be just redundant in the model). For this reason, we conducted a model selection analysis by testing all the possible combinations of predictors with different stratification and clustering. This was designed to allow for identification of the effect of CPAP intervention within the best fitting model. The AIC is recommended for small sample size and when the number of parameters among the compared models are different. The model with the lowest AIC value is usually considered to be the best data fitting model. ${ }^{14}$ Due to computational issues (all possible combinations of 25 variables produces more than 33 million models) and due to the small sample size, we restricted the model selection to bivariate interaction terms only (ie, excluded interactions between three or more predictors).

The outcome was the proportion of patients surviving at time $\mathrm{t}$, which is the time calculated from the date of COVID-19 test result and censored at 11 June 2020. The predictors were CPAP intervention (yes/no: coded 1/0),
Table 1 Baseline characteristics of the patients in the case and control groups

\begin{tabular}{|c|c|c|}
\hline & $\begin{array}{l}\text { Early group } \\
\text { (controls) } \\
\mathrm{n}=103\end{array}$ & $\begin{array}{l}\text { Late group } \\
\text { (cases) } n=103\end{array}$ \\
\hline CPAP (count) & $\begin{array}{l}3 \text { ( } 1 \text { survived } \\
\text { and two died) }\end{array}$ & $\begin{array}{l}15 \text { ( } 8 \text { survived } \\
\text { and seven died) }\end{array}$ \\
\hline $\begin{array}{l}\text { Mean A-a gradient at the start of } \\
\text { CPAP }\end{array}$ & 40.89 & 40.83 \\
\hline $\begin{array}{l}\text { Mean A-a gradient at the end of } \\
\text { CPAP }\end{array}$ & 33.90 & 31.94 \\
\hline Mean A-a difference start/end & 19.53 & 7.03 \\
\hline Median $\mathrm{O}_{2}$ for CPAP patients $(\mathrm{kPa})$ & NA & 9.4 \\
\hline $\begin{array}{l}\text { Median } \mathrm{CO}_{2} \text { for CPAP patients } \\
(\mathrm{kPa})\end{array}$ & NA & 4.8 \\
\hline Median $\mathrm{FiO}_{2}$ for CPAP patients & NA & 56.5 \\
\hline Intubated (count) & 15 & 7 \\
\hline $\begin{array}{l}\text { Average stay in hospital (until } \\
\text { discharged) (days) }\end{array}$ & 8 & 12 \\
\hline $\begin{array}{l}\text { Mean time in hospital until death } \\
\text { (days) }\end{array}$ & 9.2 & 7 \\
\hline Average age (years) & 70.5 & 70.3 \\
\hline Average age at death (years) & 74.9 & 76.1 \\
\hline Sex (count) & $66 \mathrm{M} / 37 \mathrm{~F}$ & $66 \mathrm{M} / 37 \mathrm{~F}$ \\
\hline Deaths (count) & 41 (34M/7F) & $46(35 \mathrm{M} / 11 \mathrm{~F})$ \\
\hline From care homes (count) & 21 & 39 \\
\hline Diabetic (count) & 23 & 35 \\
\hline Pulmonary (count) & 35 & 22 \\
\hline With asthma & 14 & 10 \\
\hline With emphysema & 20 & 9 \\
\hline With bronchiectasis & 3 & 5 \\
\hline Cardiac (count) & 36 & 27 \\
\hline Renal (count) & 10 & 7 \\
\hline Hypertension (count) & 36 & 36 \\
\hline $\begin{array}{l}\text { Average no of comorbidities per } \\
\text { patient }\end{array}$ & 1.37 & 1.23 \\
\hline
\end{tabular}

A-A stands for alveolar-arterial gradient a measure of gas/ exchange diffusion. Values of $\mathrm{O} 2, \mathrm{CO} 2$ and $\mathrm{FiO} 2$ for early group not reported since they were available for only one patient. *Since confirmation of COVID-19.

CPAP, continuous positive air pressure; Fio2, fractional inspired oxygen; ICU, Intensive Care Unit; NA, not available.

intubated (yes/no: coded 1/0), A-a gradient (continuous variable), Age (continuous variable), pulmonary comorbidity (yes/no: coded $1 / 0$ ), renal comorbidity (yes/no: coded $1 / 0$ ), cardiac comorbidity (yes/no: coded $1 / 0$ ), diabetic comorbidity (yes/no: coded $1 / 0$ ) and hypertension comorbidity (yes/no: coded 1/0). Within the pulmonary comorbidity domain, we identified asthma (yes/no: coded 1/0), emphysema (yes/no: coded 1/0) and bronchiectasis (yes/no: coded 1/0). Residency in care homes was also included (yes/no: coded $1 / 0$ ). We stratified the Cox proportional hazard model (which allows for non-proportional hazard in the predictors) 
with candidate variables for stratification as sex (male/ female: coded 0/1), Age above 60 (below 60/above 60: coded $0 / 1$ ), number of comorbidities (taking values from 0 to 4 , the maximum number of comorbidities experienced by a single patient). Finally, we included the difference in management (early group: control vs late group: cases) by clustering the data by group (case/ control: coded $1 / 0$ ). The result is a marginal proportional hazard model fitted via generalised estimating equation method, which accounts for correlation within the cluster and within response, and HRs are adjusted to account for these correlations. Each predictor was tested for non-proportionality via Schoenfeld proportional hazard test. ${ }^{15}$

As described above, the cases and controls referred to clinical management, and therefore, cases and controls were not 'CPAP versus non-CPAP patients' but the group of patients hospitalised before and after the management decision to increase the use of CPAP treatment on COVID-19 patients.

Additional analyses were performed on only the CPAP patients. First, the Cox proportional hazard model was applied on the survival of the CPAP patients with A-a gradient as additional predictor (as three predictors: starting A-a gradient, ending A-a gradient and their differences). Finally, the Kaplan-Meier plot of the CPAP patients survival probability versus the time when the CPAP was initiated since hospital admission was calculated.

All statistical analyses are performed in R-cran software (V.3.6.2) with packages survival (stratified CPH for clustered data, survival probability estimation and proportional hazard test), survMisc (for adjusted r-square), MuMIn (model selection and averaging) and AICcmodavg (for AIC calculation for CPH models).

\section{RESULTS}

Despite an apparent increase in the number of deaths in the late group, the lower survival time and the longer discharge time demonstrated that more severe cases were admitted later in the epidemic (table 1). The average age at death was similar in the two groups (difference of 1.2 years) and above 70 years old. Most were men, while the increase in female deaths in the late group is not statistically significant $(\mathrm{p}=0.6$ for two sample test for equality of proportions with continuity correction). The significant decrease in the A-a gradient in the patients who have had CPAP is indicative of the profound physiological improvement in diffusion after CPAP application which results in improved ventilation and consequently survival.

Finally, an increase in patients from care homes was recorded later in the outbreak (20\% in the controlsearly group, and $38 \%$ in the cases-later group) (table 1). This finding echoes the increasing national concern for care home residents during the pandemic and has led to marked revisions of policy which was originally published on 2 April 2020 and recently revised. ${ }^{16}$

In terms of comorbidities, figure 1 shows the differences in mortality as proportion of people died with a specific comorbidity or a combination of these in the

\section{Early group (control)}

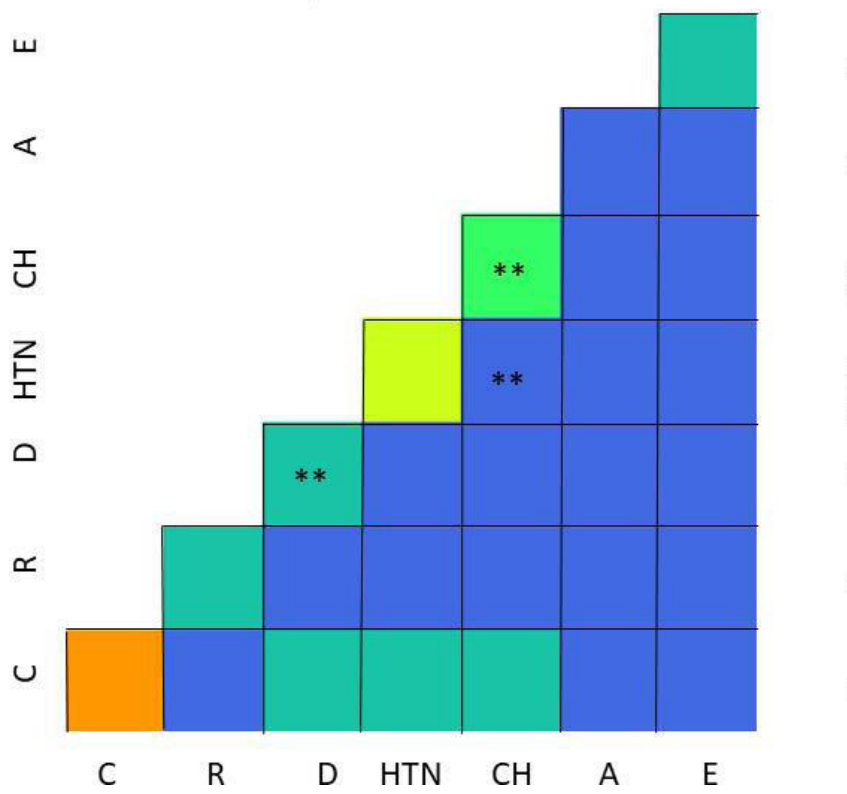

\section{Late group (cases)}

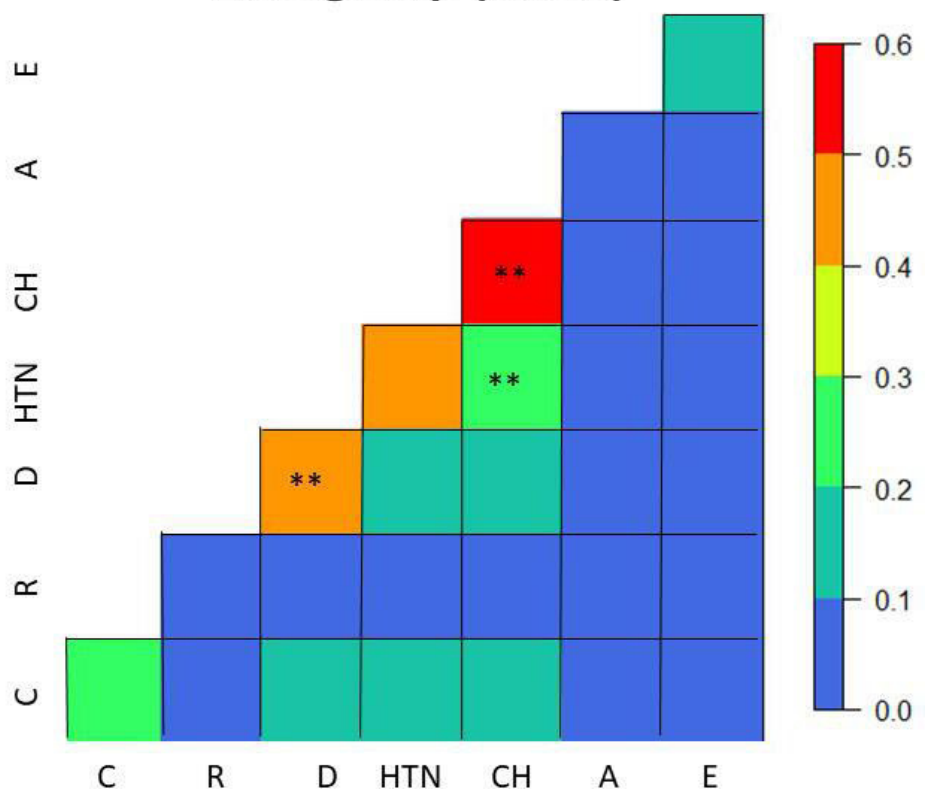

Figure 1 Proportion of deaths per comorbidity (diagonal elements) and their combinations (off-diagonal elements) in the early group (left) and later group (right). The ${ }^{* *}$ symbol indicates that the proportions of that comorbidity or combination of comorbidities between early and late group is statistically significant $(p<0.05$ for two sample test for equality of proportions with continuity correction). Bronchiectasis not included due to the low numbers. $\mathrm{A}$, asthma, $\mathrm{C}$, cardiac; $\mathrm{CH}$, patient from care homes; D, diabetes; E, emphysema; HTN, hypertension; R, renal; 


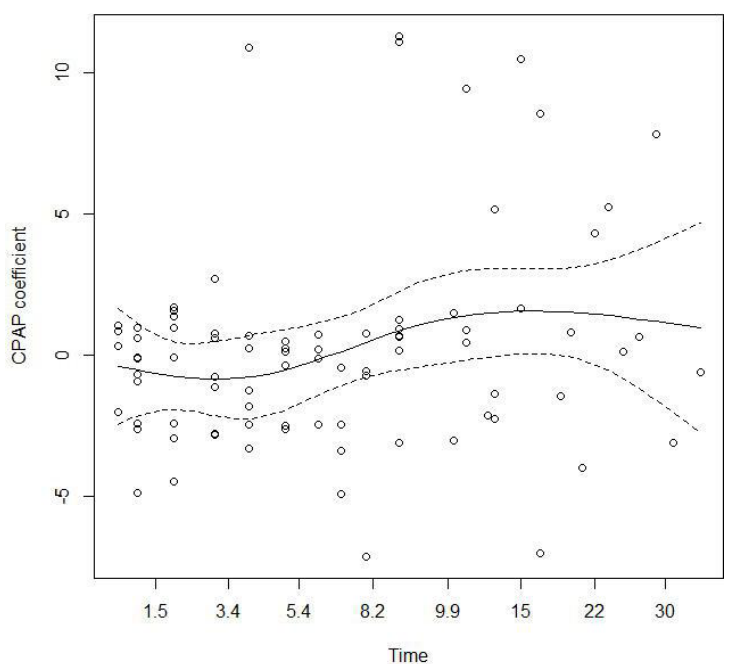

Figure 2 Proportional hazard test. The plot shows the Schoenfeld residuals which are not independent on time, as evident from the smoothing spline (continuous line). Dashed line represents the $95 \% \mathrm{Cl}$ around the spline. CPAP, continuous positive air pressure.

two groups. There are several changes between the early and late group, but only three are statistically significant: diabetes, increasing from $19 \%$ to $43 \%$ of deaths in the early and late group, respectively; patients coming from care homes, increasing from $27 \%$ to $52 \%$; and those coming from care homes with hypertension, increasing from $8 \%$ to $26 \%$. Interestingly, pulmonary comorbidities, such as asthma and emphysema had similar low proportions of death rates between the two groups (figure 1).

Although the mortality in patients treated with CPAP is lower in the late group, the number of CPAP patients in the early group is too small for a direct comparison. The effect of CPAP was then evaluated by modelling the data with an SCPHC.

We have compared and ranked 8388608 models. The best model for lowest AIC and largest Akaike weight included CPAP, sex stratified, age, intubation, hypertension and residency in care homes. The data were clustered by group (early or late). This model showed a fail in the assumption of proportionality by the CPAP variable $(\mathrm{p}=0.0128)$ (figure 2).

To correct for the non-proportionality, we have stratified the model by CPAP and time, considering a set of different time lags (below/above $2,3,4, \ldots, 15$ days). As a reminder of context, time is the time spent in hospital by a COVID-19 patient starting on the result day of the COVID-19 test and ending with death or discharge of the patient. The best fit was obtained with a stratification of CPAP in two strata: below or equal to 7 days and above 7 days of time $t$ ( $\mathrm{p}=0.44$ for the proportional hazard test). Based on this stratification we named CPAP applied to patients with short stay in the hospital, that is 7 days or less, as 'early CPAP'; and CPAP applied to patients with long stay in the hospital, that is, more than 7 days, as 'late CPAP'.
Table 2 Summary results for the stratified mixed effect Cox proportional hazards model

\begin{tabular}{llll}
\hline Variable & Reference & HR & $\mathbf{9 5 \%} \mathbf{C l}$ \\
\hline Age & & 1.03 & 1.02 to 1.04 \\
Hypertension & 0 & 1.72 & 1.46 to 2.01 \\
\hline $\begin{array}{l}\text { Intubated } \\
\text { From care home }\end{array}$ & 0 & 2.86 & 1.29 to 6.36 \\
$\begin{array}{l}\text { CPAP stratified by } \\
\text { time } \leq 7 \text { days }\end{array}$ & & 1.96 & 1.01 to 3.80 \\
$\begin{array}{l}\text { CPAP stratified by } \\
\text { time }>7 \text { days }\end{array}$ & & 0.38 & 0.36 to 0.40 \\
$\begin{array}{l}\text { Interaction } \\
\text { hypertension and } \\
\text { intubated }\end{array}$ & $0: 0$ & 0.23 & 0.11 to 0.45 \\
\hline
\end{tabular}

CPAP, continuous positive air pressure.

From the selected model it is shown that CPAP adopted in patients with short hospitalisation (within a week from the time of the COVID-19 test results) is associated with a decreased risk of death, although where the CPAP is adopted in patients with longer hospitalisation (more than a week) is associated with increased death rate (table 2). Intubation, age, hypertension, residency in care homes are associated with increased risk of death while the interaction between hypertension and intubation is associated with lower risk of death.

The model with CPAP has a larger log likelihood than the same model without CPAP, for example, with age, intubation, hypertension and care homes as predictors and sex as strata (anova test, $\mathrm{p}<0.0001$ ), showing the improvement in model fitting when stratified CPAP is included as predictor. The overall r-squared was 0.3.

The extreme (highest and lowest) predicted survival probabilities for a set of predictor combinations are provided in table 3 (full Table in online supplemental file 2). It is shown that CPAP with hospital stay of 7 days or less for a female patient at her 60 without hypertension and not resident in care home increases survival by $10 \%$ compared with the same patient who did not receive CPAP.

The gas/exchange diffusion (A-a gradient) (table 1) at the start of the CPAP is available for all the CPAP patients. The survival of the CPAP patients was found to increase with higher A-a gradient at the start of the CPAP (HR $1.011 ; 95 \%$ CI 1.010 to 1.013). In figure 3 is reported the Kaplan-Meier survival curve of CPAP patients based on the time of CPAP from the day of hospital admission.

Figure 3 shows that when CPAP is commenced at 4 days or fewer from admission to hospital the survival probabilities are above $73 \%$ (95\% CI 53\% to 99\%) with a maximum of $87 \%$ for 1 day (95\% CI $73 \%$ to $100 \%$ ). There is a sharp decrease from 4 to 5 days with survival probability down to $55 \%$.

Finally, we also compared the CI of the coefficients of the predictors in the selected model with the confidence 


\begin{tabular}{|c|c|c|c|c|c|c|c|c|}
\hline CPAP & Age & Sex & Hypertension & $\begin{array}{l}\text { From care } \\
\text { home }\end{array}$ & Intubated & Time & Group & $\begin{array}{l}\text { Survival } \\
\text { probabilities }\end{array}$ \\
\hline Yes & 60 & Female & No & No & No & $\leq 7$ days & Early & 0.91 \\
\hline Yes & 60 & Female & No & No & No & $\leq 7$ days & Late & 0.91 \\
\hline Yes & 60 & Female & Yes & No & Yes & $\leq 7$ days & Early & 0.89 \\
\hline Yes & 60 & Female & Yes & No & Yes & $\leq 7$ days & Late & 0.89 \\
\hline \multicolumn{9}{|c|}{...See online supplemental files for values in between... } \\
\hline No & 80 & Male & No & Yes & Yes & $\leq 7$ days & Early & 0.00 \\
\hline No & 80 & Male & No & Yes & Yes & $\leq 7$ days & Late & 0.00 \\
\hline Yes & 80 & Male & No & Yes & Yes & $>7$ days & Early & 0.00 \\
\hline Yes & 80 & Male & No & Yes & Yes & $>7$ days & Late & 0.00 \\
\hline
\end{tabular}

Only the top four and bottom four are shown. We considered only two ages 60 and 80 years online supplemental file 2 .

CPAP, continuous positive air pressure.

intervals of the predictors of an averaged model (considering the top 5\% models produced during model exploration) as described in literature. ${ }^{14}$ The model averaging using all the possible combinations of predictors and strata confirmed the results shown in table 2 but with wider, confidence intervals (table 4). In averaging all the models an additional four predictor interactions became important: emphysema, asthma, renal and diabetic comorbidities. It is important to note that the significance of these predictors in the averaged model but not in the best fitting model is an indication of a weak (but significant) effect of this variables, that therefore cannot be excluded in future analyses.

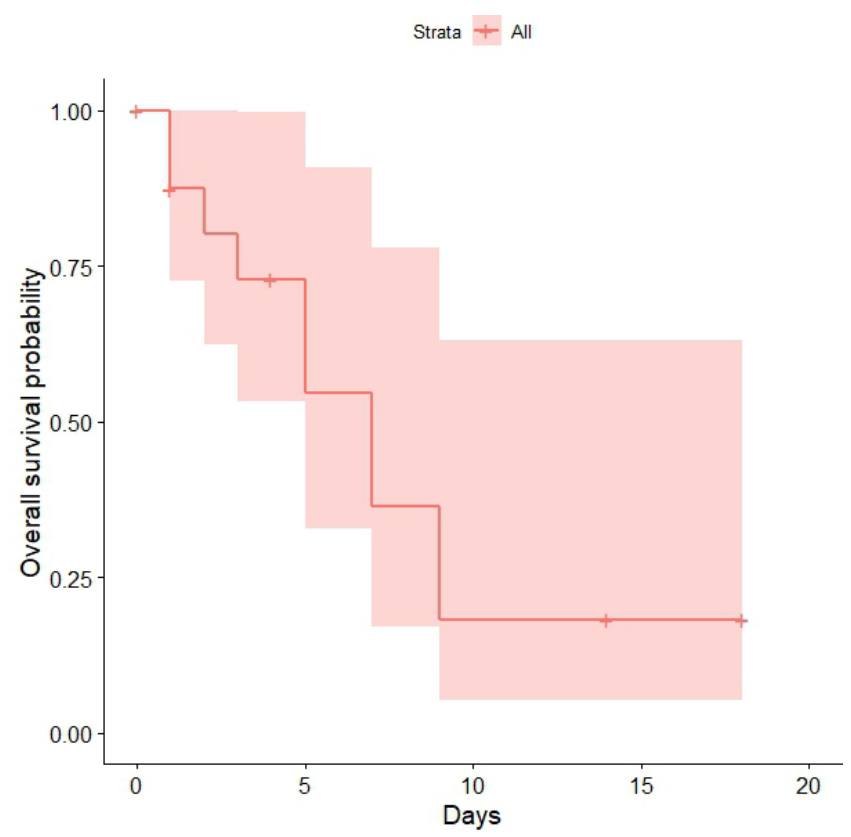

Figure 3 Survival probability of CPAP patients. The days refer to the time the CPAP is performed since hospital admission. therefore, performing the CPAP in the first days of hospital admission is associated with large survival probabilities. CPAP, continuous positive air pressure.

\section{DISCUSSION}

This evaluation describes how the clinical teams caring for COVID-19 patients altered their strategy by introducing the use of CPAP during the outbreak and with growing confidence identified that earlier use may produce improvement in outcome. At the start of the pandemic, CPAP was considered to be a rescue treatment. It was considered that it might stop some patients needing ventilation in an ICU. As clinical expertise developed, enhanced by clinical knowledge derived from CT scanning, the teams reformed their strategy by using CPAP earlier in the management pathway. This was underpinned by knowledge that early non-invasive ventilator pressure support with oxygen therapy augment recruitment, potentially preventing high pressure induced lung injury, reabsorption atelectasis and consequently reducing the tissue stress, vascular flow and fluid leakage. ${ }^{17}$ These pathophysiological changes if prevented

Table $495 \%$ Cls for the HRs of the predictors of the averaged model

\begin{tabular}{lll}
\hline Predictors (or interactions) & $\mathbf{2 . 5 0 \%}$ & $\mathbf{9 7 . 5 0 \%}$ \\
\hline Age & 1.01 & 1.05 \\
\hline CPAP stratified by time $>$ 7 days & 1.19 & 3.77 \\
\hline Emphysema:intubated & 1.21 & 3.42 \\
\hline From care home:asthma & 1.60 & 21.53 \\
Hyperthension & 1.08 & 2.29 \\
\hline Intubated & 1.04 & 5.44 \\
CPAP stratified by time $\leq 7$ days & 0.21 & 0.51 \\
Diabetic:asthma & 0.16 & 0.87 \\
Emphysema:renal & 0.17 & 0.78 \\
\hline From care home:diabetic & 0.13 & 0.79 \\
\hline Hyperthension:intubated & 0.10 & 0.60 \\
\hline
\end{tabular}

Only significant HRs are shown. Full table available in online supplemental file 3 .

COPD, Chronic Obstructive Pulmonary Disease; CPAP, continuous positive air pressure. 
early ensure the work of breathing remains low obviating the need for invasive ventilation in some cases.

It is this divergence in strategy which is pivotal in understanding the significance of the findings from this evaluation. Use of CPAP early in the course of a patient's admission is associated with reduced mortality, whereas use of CPAP with patients with long stay in hospital is associated with increased mortality as confirmed by the results of both the analysis of the full cohort of patients (SCPHC model) and the CPAP patients only (KaplanMeier survival curve). It is the advocation of earlier CPAP use to help to prevent further clinical deterioration that is the strategy that this evaluation suggests. To date there remains a paucity of emergent evidence for efficacy of CPAP for treating COVID-19. Encouragingly, within the UK (April 2020) a positive case series review of 24 patients who received CPAP treatment, demonstrated that $58 \%$ of patients avoided mechanical ventilation and that there was a remarkable $79 \%$ survival rate. ${ }^{18}$ Additionally, beneficial CPAP impact on prognosis has been demonstrated where non-responders had a double increase in mortality when compared with responders. ${ }^{19}$ On reflection and as evidence emerges, our experiences in developing care pathways in the treatment of COVID-19 have potentially been mirrored throughout the UK and internationally. ${ }^{20} 21$

While there are limitations to the present evaluation, the work summarises early clinically based interventions delivered with 'best endeavours'. It establishes the clinical view that CPAP has the potential to be an effective treatment option although the development of further strategies and studies in the future is needed. While these findings support the use of CPAP as only few other studies have reported, the other factors linked to mortality have been previously established in the literature (age in particular). ${ }^{22}$

Although the role of CPAP is the most significant clinical outcome from this work, residency in care homes is the most statistically significant risk factor. COVID-19 infections early in the Wigan outbreak included relatively few patients from care homes, but this increased as the pandemic evolved. The impact of COVID-19 on care home residents has received significant media attention during the pandemic and continues to be a concern. Since the beginning of the COVID-19 pandemic (between the period 2 March and 1 May 2020, registered up to the 9 May 2020) there were 45899 deaths of care home residents (wherever the death occurred); of these 12526 involved COVID-19, which is $27.3 \%$ of all deaths of care home residents. Of deaths involving COVID-19 among care home residents, $72.2 \%$ (9,039 deaths) occurred within a care home, and $27.5 \%$ (3444 deaths) occurred within a hospital. Of all deaths in hospital from 2 March 2020 involving COVID-19, 14.6\% were accounted for by care home residents. ${ }^{23}$ These figures could, therefore, suggest that the inability of care homes to shield vulnerable residents could have contributed to the growing admission and death rate documented in this study.
Significant policy changes are now published and have been recently revised emphasising enhanced protection, testing and shielding for vulnerable residents living in a care home setting. ${ }^{16}$

Other studies have demonstrated the importance of comorbidities in the COVID-19 associated mortality rate. We chose to include pulmonary, renal, cardiac, diabetic and hypertension co-morbidity within the analysis as emerging data demonstrated the increase risks for people with COVID-19.22 24-28 Cardiometabolic diseases, including diabetes, hypertension and cardiovascular disease have been shown to be more common that chronic pulmonary disease in COVID-19 patients and shown to correlate with increased disease severity. ${ }^{25}$ Diabetes is also now known to be a risk factor for rapid progression and worse prognosis and could lead to rapid deterioration. ${ }^{28}$ Hypertension alone is also associated with composite poorer outcomes which include mortality and may increase the risk of developing more severe COVID-19 and the need for ventilation $^{27}$ (although a recent analyses on more than 10,000 COVID-19 deaths, found hypertension as protective factor. $^{22}$

The prevalence of patients with COPD has been found to be low but it has been found elsewhere that those with severe disease have a much worse prognosis ${ }^{29}$ and the severity of the respiratory disease significantly affects both outcome and recovery. ${ }^{30}$ COPD patients are generally older potentially have multiple comorbidities which may explain poorer outcomes. The limitation of invasive mechanical ventilation options in patients with multimorbid medical conditions may also add to higher mortality. Asthma on the other hand is a disease generally affecting the younger population with the mainstay of treatment being inhaled steroids. Potentially this provides an immune-protective effect in patients affected with COVID-19 contributing to better outcomes, ${ }^{31}$ as it was found in the average model of this analysis

The analysis within this evaluation in terms of comorbidities has shown differences in mortality due to specific comorbidity or within combinations which is not unexpected. There are, however, comorbidities interactions that provided protection instead of risk (results from the averaged model in table 4), and therefore, are in need of more investigation. Those who improved on CPAP also had a lower mean A-a gradient as severe hypoxia was corrected on commencing the CPAP early which in turn facilitates improved ventilation. The most important factor in CPAP survival was the initial A-a gradient, with lower values associated to increase in survival which is a novel finding itself and that will need further studies. The early use of CPAP potentially reduces lung damage during the worst of the COVID-19 infection and allows the patient to recover from the inflammatory effects. Late use, however, functions as a rescue treatment, and while it improves the A-a ratio does not prevent lung damage thus leading to additional inflammation and a reduction in survival chances. 
Because of the emerging COVID-19 crisis, the Trust was prepared to develop new modalities of treatment and models of delivery in exceptionally rapid timescales. These are reflective of the overwhelming nature of the outbreak but are also relevant to other countries as the outbreak continues to evolve. Whist the Northern Hemisphere was initially subjected to the largest outbreaks during early 2020, the Southern Hemisphere has proven to have the larger outbreaks during the middle of 2020 as the seasons change and winter arrived in the South. ${ }^{32}$ Healthcare in much of the Southern Hemisphere has lower investment and Intensive Care facilities are less well established. They were more likely to be overwhelmed. ${ }^{33}$ The need for a low cost, simple respiratory support measure is likely to prove even more important as the Pandemic continues to evolve. There are emerging calls from scientists for evidence on the role of CPAP in COVID- $19,{ }^{15}$ to which this study potentially provides part of the answer.

This study is a step towards the ascertainment of the validity of CPAP as an effective treatment. There are inevitable caveats in that the numbers are small and the study design is based around an evolving picture of treatment during the crisis of an outbreak (see the Limitations section). Further work can be planned more carefully in areas where there is an emerging outbreak. Randomised controlled trials would help to establish the relative benefits of CPAP against other treatment options. Results are still awaited from the RECOVERY RS study in the UK which compares the effectiveness of three ventilation methods; CPAP, HFNO and standard care (oxygen delivered via a normal face mask/tubes).

It is likely that treatment of COVID-19-associated respiratory failure will be necessary for the foreseeable future. Planning the response to that needs to establish the most effective, efficient treatment options and is a global priority.

\section{CONCLUSION AND CALL FOR FURTHER STUDIES}

Treatment of COVID-19 has been based on anecdotal experience and pre-existing experience of other conditions. The use of CPAP has been advocated, but evidence for its benefit in COVID-19 pneumonia was lacking. This work builds on a previous audit ${ }^{12}$ showing a positive outcome (reduced mortality) in COVID-19 patients when treated with CPAP in the early days after hospitalisation. Due to the size of the Trust we were not able to further validate these findings on a larger sample size, and therefore, we hope that this work will inspire other groups and researchers to quantitatively evaluate the use of CPAP for COVID-19 pneumonia. As described above, this work can be especially valuable in low economic settings where ICU free spaces are a rarity and economic solutions are preferred; but also in modified settings. We would suggest that non-invasive early intervention using CPAP can be a key control strategy until an effective vaccine or drug therapy is found.

\section{Limitations of this study}

Readers need to take particular care in the interpretation of the HRs and survival probabilities of this work and their translation to evidence. In fact they are affected by small sample size (18 CPAP on 206 patients) and a relative low amount of variance explained by the model. To encompass these important limitations, we have applied robust statistics (stratified and clustered models; averaging millions of models to establish which variables are really important) that allowed an effective analyses of a small data set (18 CPAP cases). Therefore although some confidence intervals remain large, the directionality of the effects/associations is shown robust (CPAP within a week of admittance decreases risk of death) since most of the models we have tested found this significant association.

During the outbreak, there were other changes in management of patients notably with the opportunity to be involved in the national RECOVERY trial (https:// www.recoverytrial.net). This afforded patients the opportunity to receive medications beyond standard care. There were also changes in the management of anticoagulation during the time period. Finally, not all the potential predictors were included, such as ethnicity, other therapies and so forth.

Acknowledgements The outstanding contributions made by all members of our Multidisciplinary and Critical Care Outreach teams have been instrumental in introducing and developing this treatment intervention for our patients. Without their willingness to take this leap in faith adapting, learning and sharing their expertise, this improvement in care would not have been possible.The authors would also like to thank Steve Milan (Lancaster University) for his networking support in this study.

Contributors MF, RS and AA conceived of the study. JM contributed to refinement of the study protocol. AU collected and validated the data. KK assisted with data collection. LS analysed the data. LS and MF interpreted the statistical results. MF, JM and LS wrote the initial draft. All the authors discussed the results and have edited and approved the final manuscript. The corresponding authors attest that all listed authors meet authorship criteria and that no others meeting the criteria have been omitted.

Funding The authors have not declared a specific grant for this research from any funding agency in the public, commercial or not-for-profit sectors.

Competing interests None declared.

Patient consent for publication Not required.

Ethics approval A Service Evaluation application along with a protocol for this project was submitted to the Research and Development Department of the Trust. Permission was granted to undertake the evaluation (Local ID: RD62)

Provenance and peer review Not commissioned; externally peer reviewed.

Data availability statement Data are available on reasonable request. Data are available on reasonable request to the authors.

Supplemental material This content has been supplied by the author(s). It has not been vetted by BMJ Publishing Group Limited (BMJ) and may not have been peer-reviewed. Any opinions or recommendations discussed are solely those of the author(s) and are not endorsed by BMJ. BMJ disclaims all liability and responsibility arising from any reliance placed on the content. Where the content includes any translated material, BMJ does not warrant the accuracy and reliability of the translations (including but not limited to local regulations, clinical guidelines, terminology, drug names and drug dosages), and is not responsible for any error and/or omissions arising from translation and adaptation or otherwise.

Open access This is an open access article distributed in accordance with the Creative Commons Attribution Non Commercial (CC BY-NC 4.0) license, which permits others to distribute, remix, adapt, build upon this work non-commercially, and license their derivative works on different terms, provided the original work is 
properly cited, appropriate credit is given, any changes made indicated, and the use is non-commercial. See: http://creativecommons.org/licenses/by-nc/4.0/.

ORCID iD

Martin Farrier http://orcid.org/0000-0002-7556-6848

\section{REFERENCES}

1 Huang C, Wang Y, Li X, et al. Clinical features of patients infected with 2019 novel coronavirus in Wuhan, China. Lancet 2020.

2 Wang D, Hu B, Hu C, et al. Clinical characteristics of 138 hospitalized patients with 2019 novel coronavirus-infected pneumonia in Wuhan, China. JAMA 2020;323:10619

3 Williams TA, Finn J, Perkins GD, et al. Prehospital continuous positive airway pressure for acute respiratory failure: a systematic review and meta-analysis. Prehosp Emerg Care 2013;17:261-73.

4 Bakke SA, Botker MT, Riddervold IS, et al. Continuous positive airway pressure and noninvasive ventilation in prehospital treatment of patients with acute respiratory failure: a systematic review of controlled studies. Scand J Trauma Resusc Emerg Med 2014;22:69.

5 Faria DAS, da Silva EMK, Atallah Álvaro N, et al. Noninvasive positive pressure ventilation for acute respiratory failure following upper abdominal surgery. Cochrane Database Syst Rev 2015:CD009134.

6 Aliberti S, Radovanovic D, Billi F, et al. Helmet CPAP treatment in patients with COVID-19 pneumonia: a multicentre cohort study. Eur Respir J 2020:56:01935-2020

7 NHS England. Guidance for the role and use of non-invasive respiratory support in adult patients with COVID19 (confirmed or suspected), 2020. Available: https://www. england. nhs. uk/ coronavirus/ wp- content/ uploads/ sites/ 52/ 2020/ 03/ specialtyguide- NIVrespiratory- support- and- coronavirus- v3.pdf [Accessed 4 May 2020].

8 Rochwerg B, Granton D, Wang DX, et al. High flow nasal cannula compared with conventional oxygen therapy for acute hypoxemic respiratory failure: a systematic review and meta-analysis. Intensive Care Med 2019;45:563-72.

9 Critical care committee of Chinese Association of Chest Physician, Respiratory and critical care group of Chinese Thoracic Society, Respiratory care group of Chinese Thoracic Society. [Conventional respiratory support therapy for Severe Acute Respiratory Infections (SARI): Clinical indications and nosocomial infection prevention and control]. Zhonghua Jie He He Hu Xi Za Zhi 2020;43:189-94.

$10 \mathrm{Wu} Z$, McGoogan JM. Characteristics of and Important Lessons From the Coronavirus Disease 2019 (COVID-19) Outbreak in China: Summary of a Report of 72314 Cases From the Chinese Center for Disease Control and Prevention. JAMA 2020;323:1239-42.

11 Giwa A, Desai A, Duca AN. Coronavirus SARS-CoV-2 (COVID-19): an overview for emergency clinicians.V17.Emergency medicine practice, 2019. Available: www.ebmedicine.net

12 Ashish A, Unsworth A, Martindale J, et al. Early CPAP reduced mortality in covid-19 patients. audit results from Wrightington, Wigan and Leigh teaching hospitals NHS Foundation trust. medRxiv 2020.

13 Sugiura N. Further analysts of the data by akaike' s information criterion and the finite corrections. Commun Stat Theory Methods 1978;7:13-26.

14 Burnham KP, Anderson DR. Model selection and multimodel inference: a practical information-theoretic approach. 2nd ed. Springer-Verlag, 2002.

15 Grambsch PM, Therneau TM. Proportional hazards tests and diagnostics based on weighted residuals. Biometrika 1994:81:515-26.
16 Gov UK. DOH and social care, public health England, social admission and care of residents in a care home during COVID-19 version 2, 2020. Available: https://assets.publishing.service.gov. uk/government/uploads/system/uploads/attachment_data/file/ 914224/covid-19-admission-and-care-of-residents-in-a-care-homeguidance.pdf

17 Guan L, Zhou L, Le Grange JM, et al. Non-Invasive ventilation in the treatment of early hypoxemic respiratory failure caused by COVID-19: considering nasal CPAP as the first choice. Crit Care 2020;24:333

18 Nightingale R, Nwosu N, Kutubudin F, et al. Is continuous positive airway pressure (CPAP) a new standard of care for type 1 respiratory failure in COVID-19 patients? A retrospective observational study of a dedicated COVID-19 CPAP service. BMJ Open Respir Res 2020;7:e000639.

19 Pagano A, Porta G, Bosso G, et al. Non-Invasive CPAP in mild and moderate ARDS secondary to SARS-CoV-2. Respir Physiol Neurobiol 2020;280:103489.

20 Giwa A, Desai A, Duca AN. Coronavirus SARS-CoV-2 (COVID-19): an overview for emergency clinicians. Emergency Medicine Practice $2019 ; 17$.

21 Simonelli C, Paneroni M, Fokom AG, et al. How the COVID-19 infection tsunami revolutionized the work of respiratory physiotherapists: an experience from northern Italy. Monaldi Arch Chest Dis 2020;90:1085.

22 Williamson EJ, Walker AJ, Bhaskaran K, et al. Factors associated with COVID-19-related death using OpenSAFELY. Nature 2020:584:430-6.

23 Gov UK. COVID-19: number of outbreaks in care homes management information. Available: https://www.gov.uk/ government/statistical-data-sets/covid-19-number-of-outbreaks-incare-homes-management-information

24 Wang B, Li R, Lu Z, et al. Does comorbidity increase the risk of patients with COVID-19: evidence from meta-analysis. Aging 2020:12:6049-57.

25 Yahia F, Zakhama L, Ben Abdelaziz A. COVID-19 and cardiovascular diseases. scoping review study. Tunis Med 2020;98:283-94.

26 Liu H, Chen S, Liu M, et al. Comorbid chronic diseases are strongly correlated with disease severity among COVID-19 patients: a systematic review and meta-analysis. Aging Dis 2020;11:668-78.

27 Pranata R, Lim MA, Huang I, et al. Hypertension is associated with increased mortality and severity of disease in COVID-19 pneumonia: a systematic review, meta-analysis and meta-regression. J Renin Angiotensin Aldosterone Syst 2020;21:147032032092689.

28 Guo W, Li M, Dong Y, et al. Diabetes is a risk factor for the progression and prognosis of COVID-19. Diabetes Metab Res Rev 2020:e3319.

29 Alqahtani JS, Oyelade T, Aldhahir AM, et al. Prevalence, severity and mortality associated with COPD and smoking in patients with COVID-19: a rapid systematic review and meta-analysis. PLoS One 2020;15:e0233147.

30 Zhang J, Wang X, Jia X, et al. Risk factors for disease severity, unimprovement, and mortality in COVID-19 patients in Wuhan, China. Clin Microbiol Infect 2020;26:767-72.

31 Carli G, Cecchi L, Stebbing J, et al. Is asthma protective against COVID-19? Allergy 2020;148.

32 Scafetta N. Distribution of the SARS-CoV-2 pandemic and its monthly forecast based on seasonal climate patterns. Int J Environ Res Public Health 2020;17:3493.

33 Hopman J, Allegranzi B, Mehtar S. Managing COVID-19 in low- and middle-income countries. JAMA 2020;323:1549-50. 